\title{
Research Progress of Small Molecule Targeted Anti-tum or Drugs on "Internet+" Times
}

\author{
Lin Yan, Qiuyun Wang and Qinyuan Cheng \\ Wuxi Higher Health Vocational Technology School, Wuxi, Jiangsu 214000, China \\ paperiset@163.com
}

Keywords: Kinase inhibiter, target, small molecule, anti-tum or drug.

\begin{abstract}
At present, the molecular targeted small molecule anti-tum or drug becomes hot topic of domestic and foreign research and development of innovative drugs. There are always new products having been launched, and hundreds of products are in the clinical development stage in recent years. In this paper, we introduced some hat anti-tum or targets, and listed the representative drugs of these targets. With the development of bioscience and pharmacy, more and more effective target will be found, and more effective but less toxic compounds will be produced to provide a new approach for the treatment of tum or in the future.
\end{abstract}

\section{Introduction}

With the continuous growth of human life, malignant cancer on human health is also more and more. According to the statistics on the mortality and cause of death of major urban and rural residents in 2015, malignancy has become the leading cause of death for urban and rural residents in China [1]. At present, the main methods of treatment of malignant tumors are surgical resection, drug chemotherapy, radiation therapy, etc., and because most of the malignant tumor patients have been found in the tumor metastasis, so chemotherapy is one of the essential ways [2]. The traditional chemotherapy drugs mainly through the inhibition of splitting active cells in the DNA synthesis to achieve the purpose of killing cancer cells, but because of this method is not specific, easy to produce different degrees of normal cells, so the traditional chemotherapy drugs have strong cells Toxicity and side effects, and cancer cells are susceptible to drug resistance. In recent years, with the development of the mechanism of tumor more in-depth [3], human beings have gradually realized that the essence of the tumor is a genetic disease, is due to normal cell signaling disorders caused by cell proliferation.

With the continuous progress of tumor research, targeted therapy has gradually become a research and development of anti-cancer drug hot spots. Unlike traditional cytotoxic drugs, small molecule targeting drugs are mostly specific for membrane receptors, cell signaling pathways, cell cycle regulators, and important proteins or factors involved in angiogenesis, with relative specificity, Cell toxicity is relatively light, and has not easy to produce drug resistance, high selectivity, the dosage is relatively small and so on. Since the first kind of drug imagine maculate in 2001 by the US Food and Drug Administration approved the listing, small molecule targeting anti-tumor drugs has become the mainstream of anti-tumor drug research and development, there are hundreds of products Is in the clinical research and development stage, in recent years has also been a new product market, according to statistics, 2010-2014 a total of 19 kinds of drugs by the US Food and Drug Administration (FDA) approved the listing [4, 5]. With the development of life sciences and pharmacy, the future will find more and more effective targets and better efficacy, less toxic compounds, for cancer treatment to provide a new way.

\section{Small Molecule Targeted}

Internet+ times. Internet becomes one of the most important means for people to obtain health and medical information. It is often a first step for people to check basic information on diseases, and medicine and the searched results are often helpful to users. Among various Internet search engines, 
Google is the most widely used. More and more people use Internet search engines, especially Google, to learn diseases and possible treatments. Furthermore, more and more clinicians began to use search engine to help diagnosis. There are some studies suggests that non-physician may also occasionally obtain correct diagnoses through Internet-based search and this may help physician-patient interaction. All these studies indicate that search engines like Google could play an important role in obtaining and assessing medical information for professionals and lay people. In order to check the quality and effectiveness of search engine, some researchers have done a lot of study works. Some of their mechanism was too general and the systematic data didn't build up. Some researchers' studies were too systematic and didn't consider user's experience. Thus, it is more important for the search engines to meet public needs based on users' experience.

Drugs and Experiment. PF-03084014 was provided to the Pediatric Preclinical Testing Program by Pfizer, through the Cancer Therapy Evaluation Program (NCI). Drug was formulated in 0.5\% methylcellulose, in sterile water for injection, and stored in the dark at 40C for up to 1 month. PF-03084014 was administered orally (P.O.) at $150 \mathrm{mg} / \mathrm{kg}(112.5 \mathrm{mg} / \mathrm{kg}$ for the ALL xenografts based on toxicity testing in NOD mice) using a twice-daily schedule (Days 1-7 and 15-21) for one cycle, followed by 3 weeks of observation. PF-03084014 was provided to each consortium investigator in coded vials for blinded testing.

\section{Result and Discuss}

PF-03084014 showed little or no effect in vitro against the PPTP cell line panel using 96-hour exposure at concentrations up to 10, M. The ICSO for inhibition of Notch signaling by PF-03084014 is in the 10 to $150 \mathrm{nM}$ range with maximal inhibition by 1.0, M [4, 20]. Therefore, the lack of in vitro activity observed for PF-03084014 against the PPTP cell line panel cannot be explained by use of insufficient concentrations to induce inhibition of Notch signaling.

Table 1 In vitro activity

\begin{tabular}{ccccc}
\hline Cell line & Histotype & IIC40(um) & Panel rIC40/line rIC40 & Ymin\% \\
\hline RD & Rhabdomyosacoma & $>10$ & 1.00 & 90 \\
Rh41 & Rhabdomyosacoma & $>10$ & 1.00 & 86 \\
Rh18 & Rhabdomyosacoma & $>10$ & 1.00 & 62 \\
Rh30 & Rhabdoid & $>10$ & 1.00 & 88 \\
BT-12 & Rhabdoid & $>10$ & 1.00 & 87 \\
CHLA-266 & Rhabdomyosacoma & $>10$ & 1.00 & 69 \\
TC-71 & Ewing sarcoma & $>10$ & 1.00 & 86 \\
CHLA-9 & Ewing sarcoma & $>10$ & 1.00 & 55 \\
CHLA-10 & Ewing sarcoma & $>10$ & 1.00 & 66 \\
CHLA-258 & Ewing sarcoma & $>10$ & 1.00 & 68 \\
SJ-GBM2 & Glioblastoma & $>10$ & 1.00 & 100 \\
NB-1643 & Neuroblastoma & $>10$ & 1.00 & 85 \\
NB-Ebc1 & Neuroblastoma & $>10$ & 1.00 & 84 \\
CHLA-90 & Neuroblastoma & $>10$ & 1.00 & 78 \\
CHLA-136 & Neuroblastoma & $>10$ & 1.00 & 82 \\
NALM-6 & All & $>10$ & 1.00 & 78 \\
COG-LI-317 & All & $>10$ & 1.00 & 85 \\
RS4; 11 & All & $>10$ & 1.00 & 76 \\
MOLT-4 & All & $>10$ & 1.00 & 82 \\
CCRF-CEM(1) & All & $>10$ & 1.00 & 81 \\
CCRF-CEM(2) & All & $>10$ & 1.00 & 64 \\
Kasumi-1 & AML & $>10$ & 1.00 & 76 \\
Karpas-299 & Alcl & $>10$ & 1.00 & 78 \\
Ramos-RAI & nhl & $>10$ & 1.00 & 54 \\
Mediam & & $>10$ & 1.00 & 90 \\
Minimum & & $>10$ & 1.00 & 75 \\
Maximum & & & 1.00 & 100 \\
\hline & & & \\
\hline
\end{tabular}


Notch pathway signaling is activated in some of the solid tumor panels, as evidenced by expression patterns of NOTCHI-4, and transcriptional targets HES1 and HEY1/HEY2. Although there is circumstantial evidence that Notch pathway inhibition may be an effective therapeutic strategy for selected childhood cancers, our results demonstrate that inhibition of this pathway through y-secretase inhibition has little impact on cancer cell survival/proliferation for models across a range of histotypes.

Table 2 Summary of In Vivo activity

\begin{tabular}{|c|c|c|c|c|}
\hline Xenograft line & Tumor type & Mediam time to event & $\mathrm{P}$ & Efs \\
\hline BT-29 & Rhabdoid & 35.7 & 0.041 & 1.4 \\
\hline Kt-16 & Rhabdoid & 11.0 & $<0.041$ & 1.8 \\
\hline Kt-14 & Rhabdoid & 17.9 & 0.733 & 1.1 \\
\hline Kt-10 & Wlims & 12.9 & 0.002 & 1.3 \\
\hline Kt-11 & Wlims & 23.4 & 0.517 & 1.1 \\
\hline Kt-13 & Ewing & 16.0 & 0.025 & 1.4 \\
\hline Sk nep -1 & Ewing & 11.6 & 0.553 & 1.2 \\
\hline Ew 5 & Ewing & 10.2 & $<0.001$ & 1.6 \\
\hline Ew 8 & Ewing & 10.6 & 0.034 & 1.6 \\
\hline Тc-71 & Ewing & 11.6 & $<0.001$ & 1.9 \\
\hline Chla & Ewing & 22.0 & 0.173 & 1.1 \\
\hline Rh10 & Ewing & 18.3 & 0.263 & 1.6 \\
\hline $\mathrm{Rh} 28$ & Alveolar rms & 14.3 & 0.966 & 1.1 \\
\hline $\mathrm{Rh} 30$ & Alveolar rms & 10.8 & 0.168 & 1.1 \\
\hline Rh30r & Alveolar rms & 12.5 & $<0.001$ & 1.5 \\
\hline Rh41 & Alveolar rms & 11.9 & 0.052 & 1.4 \\
\hline Bt-28 & Alveolar rms & 14.2 & 0.525 & 1.1 \\
\hline $\mathrm{Bt}-45$ & Medulloblastoma & 35.8 & 0.148 & 1.1 \\
\hline Bt-50 & Medulloblastoma & $>\mathrm{EP}$ & 0.647 & - \\
\hline Bt-36 & Medulloblastoma & $>\mathrm{EP}$ & 1.000 & - \\
\hline Bt-41 & Medulloblastoma & $>\mathrm{EP}$ & 1.100 & - \\
\hline GBM2 & Glioblastoma & 11.3 & 0.213 & 0.8 \\
\hline BT-39 & Glioblastoma & 11.7 & 0.027 & 1.5 \\
\hline D645 & Glioblastoma & 9.9 & 0.114 & 1.3 \\
\hline D456 & Glioblastoma & 8.5 & 0.639 & 1.3 \\
\hline NB-SD & Neuroblastoma & 11.9 & 0.622 & 1.0 \\
\hline NB-1771 & Neuroblastoma & 10.8 & 0.141 & 1.3 \\
\hline NB-1691 & Neuroblastoma & 6.9 & 0.131 & 1.1 \\
\hline NB-EBC1 & Neuroblastoma & 5.3 & 0.298 & 1.1 \\
\hline NB-1643 & Neuroblastoma & 12.3 & 0.268 & 0.9 \\
\hline OS-2 & Osteosarcoma & 19.8 & 0.047 & 1.2 \\
\hline OS-17 & Osteosarcoma & 24.3 & 0.005 & 1.3 \\
\hline OS-9 & Osteosarcoma & 18.4 & 0.042 & 1.1 \\
\hline OS-33 & Osteosarcoma & 12.6 & $<0.001$ & 1.1 \\
\hline OS-31 & Osteosarcoma & 20.6 & $<0.001$ & 1.3 \\
\hline All-2 & Osteosarcoma & 16.2 & 0.858 & \\
\hline All-4 & All b & 4.3 & 0.351 & \\
\hline All-8 & All-b & 5.0 & 1.000 & \\
\hline All-16 & All t & 23.6 & 0.567 & \\
\hline All-27 & All $t$ & 5.0 & 0.913 & \\
\hline All-29 & All $t$ & 5.0 & 0.241 & \\
\hline All-30 & All $t$ & 6.2 & 0.620 & \\
\hline All-31 & All t & 13.6 & 0.015 & \\
\hline All-31 & Tertiary & 6.9 & 0.507 & \\
\hline
\end{tabular}

It is important to point out the limitation of this study, i.e., the user experience in medical information or information obtained by patients does not necessarily match that from their doctors. In the era of patient-centered care, evidence-based practice, personalized medicine, and health care 
system reform, vast availability of health information on the Internet has a significant impact on modern medical practice. The patients should not be expected to fully understand the significance of the medical information that they searched. Given that patients deserve the best care with accurate diagnosis and the most effective treatment, it is the responsibility for medical professionals to explain the medical knowledge and terminologies in a lay language to their patients in the patient-centered decision-making process. Explanations of nature of disease, possible diagnostic procedures, therapeutic options, potential side effects and cost should be offered to their patients. Ultimately, patient's health care plan should reflect the best-available scientific evidence, the maximal benefit to each particular patient, high quality of life, and cost effective and feasible management, while Internet search results may provide a starting point for patient-doctor communication. To further foster this new trend of patient care model in the Internet age, proper evaluation of search engines for health information is critical. Our case study, although performed only in a small scale, provides the first-hand data from users in terms of their experience in using Internet to search for medical information, and is a useful attempt to evaluate the usability on the Internet search engine for obtaining medical information. To our knowledge, this is the first quantitative usability test from a software engineering perspective in applying Internet search for obtaining medical information. This study may provide some suggestions in improving the overall usability of health-related Internet search.

\section{Summary}

Malignant tumor is still the world's second largest fatal disease. Conventional treatment such as surgery, chemotherapy and radiotherapy of malignant tumor with different methods, molecular targeted therapy as a new method for tumor therapy, because of its high efficacy, low toxicity and side effects and high specificity and other advantages has become a hot research topic in the treatment of cancer. With the development of molecular biology and biotechnology industry continue to promote and support people to explore the tumor is deepening, tumor specific molecular targets have been more and more recognized by people, anti tumor small molecular target drug to subsequent development. According to the different characteristics of tumor molecular biology in this study, tumor targeting of small molecular target and the corresponding summary and review to the drug.

\section{References}

[1] Bai J, Yang C, Huang G, et al. Research Progress of Small-molecule Targeted Anti-tumor Drugs. Anti-tumor Pharmacy, Vol. 2 (2015) No. 6, pp. 56-64.

[2] Li F. Potential pharmacokinetic interactions of therapeutic cytokines or cytokine modulators on small-molecule drugs: mechanistic understanding via studies using in vitro systems : Drug Metabolism and Drug Interactions. Drug Metabolism \& Drug Interactions, Vol. 29 (2014) No. 9, pp. 17. (In Chinese)

[3] Debnath A K. Progress in identifying peptides and small-molecule inhibitors targeted to gp41 of HIV-1. Expert Opinion on Investigational Drugs, Vol. 15 (2006) No. 5, pp. 465-78. (In Chinese)

[4] Chen X Y, Zhang H, Gao C Y, et al. Discussion and case analysis of clinical development strategy of small-molecule targeted anti-cancer drugs. Chinese Journal of New Drugs, Vol. 22 (2013) No. 4, p.46-52.

[5] Liu X, Ding Z S, Jiang F S. Research progress of polyethylene glycol modified small molecule drugs. Chinese Journal of Pharmacology \& Toxicology, Vol. 24 (2010) No. 5, p. 380-386. (In Chinese).

[6] Obradovic M, Mrhar A, Kos M. Market uptake of biologic and small-molecule-targeted oncology drugs in Europe. Clinical Therapeutics, Vol. 31 (2009) No. 12, pp. 2940. 
[7] Chen X Y, Zhang H, Gao C Y, et al. Discussion and case analysis of clinical development strategy of small-molecule targeted anti-cancer drugs. Chinese Journal of New Drugs, (2013).

[8] Sonpavde G, Hutson T E, Sternberg C N. Pazopanib, a potent orally administered small-molecule multitargeted tyrosine kinase inhibitor for renal cell carcinoma. Expert Opinion on Investigational Drugs, Vol. 17 (2008) No. 2, pp. 253.

[9] Vrbanec D, Belev B. Targeted drugs in gastrointestinal oncology. Medicus, Vol. 21 (2013), pp. 249-257.

[10] Jonas B D. Anti-tamper system. US 20070168669 A1[P], (2007). 